\title{
RF PARAMETER CURVES FOR A PROTON DRIVER SYNCHROTRON
}

\author{
J. A. MacLachlan, Z. Qian, J. E. Griffin \\ Fermi National Accelerator Laboratory, Box 500, Batavia IL 60510-0500*
}

\section{INTRODUCTION}

High average beam power proton synchrotrons in the medium energy range are under consideration at several laboratories for intense and specialized secondary particle sources like muon colliders and $\nu$ factories. A $12-16 \mathrm{GeV}$ machine with a $15 \mathrm{~Hz}$ cycle and $3 \cdot 10^{13}$ p/pulse capability called the Proton Driver (PD) has been studied as a replacement for the Fermilab Booster and as a base for future facilities.[1] A staged development is proposed, initially using 20 modified $53 \mathrm{MHz}$ Booster cavities in $12 \mathrm{GeV}$ operation.[2] A second stage would allow $16 \mathrm{GeV}$ top energy using a $7.5 \mathrm{MHz}$ rf system consisting of $10015 \mathrm{kV}$ low-Q cavities.[3] This paper discusses the choices of $\mathrm{rf}$ system parameters made in the design study. The limited number of existing Booster cavities has led to consideration for stage 1 of an inductive insert in the ring to aid initial beam capture by compensating longitudinal space charge, an admittedly speculative expedient requiring followup with further calculation and some beam experiments. This report is one of nineteen papers at this conference by members of the Proton Driver design team; it relies on these others to help establish the general context.

\section{FIRST STAGE (53 MHZ RF)}

Stage 1 of the PD serves to replace the present Booster in the Fermilab injector chain and perhaps directly for low energy neutrino production. The top energy is $12 \mathrm{GeV}$ using a lattice designed for $16 \mathrm{GeV}$ capability. It will employ refurbished Booster rf cavities modified to give a 5 inch aperature. The parameters defining rf requirements are collected in Table 1.

The combination of performance demands with the mandated use of a $400 \mathrm{MeV}$ linac injector and modified Booster cavities calls for some unconventional measures. The space charge impedance corresponding to the perfectly conducting wall force is $Z_{\|} / n \approx-230 i \Omega$ at injection energy. To control the space charge defocusing, a tunable inductive insert is proposed to cancel this impedance throughout most of the cycle. The insert looks attractive in the modeling; it makes the difference between $96.8 \%$ and $99.97 \%$ for the particle transmission efficiency for the complete cycle. The idea is not new; [4] it has been tried in two different machines.[5, 6] However, studies have not been carried out over a wide range of beam energy, momentum spread, etc., and more are needed.

The magnet ramp is driven by a $15 \mathrm{~Hz}$ resonant supply plus an independant second harmonic supply that is adjusted in phase and amplitude to minimize the required peak

\footnotetext{
* Operated by the Universities Research Association under contract number DE-AC02-76CH03000 with the U. S. Department of Energy
}

rf voltage. The parameter optimization is driven primarily by the effort to minimize beam loss. Because the rf voltage limit is so strigent, loss limitation naturally relates closely to longitudinal emittance preservation also.

\subsection{Capture and Acceleration}

A macroparticle tracking model has been used for the entire cycle from multi-turn injection through matching to Main Injector buckets. The injected protons are taken as a continuous coasting beam at the energy of $B_{\text {min }}$ lasting up to $90 \mu$ s timed symmetrically about $B_{\text {min }}$; assymetric timings and energy offsets have not proved helpful. For nominal linac intensity, $70 \mu \mathrm{s}$ is sufficient to give the required $3 \cdot 10^{13}$ protons, but efficiency does remain good over a longer injection time. The perfectly conducting wall term and the inductive insert are the only sources for the collective potential in these simulations.

The rf voltage is raised linearly during injection from 0 to $65 \mathrm{kV}$. It is then raised somewhat more slowly to establish a bucket area of $0.064 \mathrm{eVs}$ at $226 \mu \mathrm{s}$. Because the slip factor $\eta$ is large at injection, the particles near $\pm 180^{\circ}$ of $\mathrm{rf}$ phase are all captured in this simple manouver. Certainly some are quite close to the separatrix and subject to later loss, but these losses are practically eliminated by the inductive insert. They could also be controled with a substantially higher rf voltage. After $226 \mu$ s, the voltage curve holds the bucket area constant until $4.96 \mathrm{~ms}$ where the voltage has reached the design limit of $1.2 \mathrm{MV}$. It is held at that value until $\eta$ has dropped sufficiently at about $30 \mathrm{~ms}$ to permit reduction. Because of decreasing $\eta$ and the control of $\dot{B}$ by a second harmonic component in the magnet current, the bucket area scarcely changes until it is allowed to rise at the end of the cycle. Nonetheless, in the absence of the inductive insert there are losses at maximum $\dot{p}$ (about 0.025 $\mathrm{s}$ into the cycle). This indicates that the $1.2 \mathrm{MV}$ peak voltage is marginal. $\dot{B}$ reaches zero at $37.93 \mathrm{~ms}$. The voltage required for acceleration alone is $1.09 \mathrm{MV}$ at maximum $\dot{p}$, so there is not much rf focusing. The synchronous phase reaches about $64^{\circ}$.

The curves for $p(t), \dot{p}(t), V_{\mathrm{rf}}(t)$, bucket area $S_{B}(t)$, and synchrotron tune $\nu_{s}(t)$ are plotted together in Fig. 1. The curves are normalized to the range between zero and one to display their qualitative interrelation; the magnitudes are indicated by the parametrs in Table 1. Fig. 2 displays the normalized rms emittance, the rms bunch width, and the rms bunch height simillarly normalized.

The apparent effectiveness of an inductive insert and its importance for low loss with the $h=126 \mathrm{rf}$ has resulted in its tentative adoption for reducing beam loss and emittance growth. A limited amount of rf focusing is suplemented 
with self-excited focusing voltage. However, the inductance will have a real impedance component which dissipates rf power and could furthermore cause self-trapping instability. Careful studies of the tradeoffs are required to establish net benefit.

The slip factor is so high at injection that the captured beam has energy-phase correlation (bunch tilt) which wastes precious bucket area and causes beam loss. Dividing the rf into two parts on opposite sides of the ring reduces the tilt and resulting loss. Dividing the rf into three equally spaced groups would make a small additional improvement. The planned configuration of the injection, extraction, and collimation systems looks inconsistent with a three-way division.

Table 2 shows the injection-to-extraction transmission efficiency and emittance at extraction for different departures from the optimum modeling result. The top entry is the best result obtained, and each entry following gives the transmission when one condition is changed without attempting to reoptimize the other parameters. Possibly some of the apparently lost efficiency could be recovered in such a reoptimization, but the intention is only to suggest the importance of various conditions to the optimum obtained. The lower final emittance for the more closely grouped cavities reflects directly the removal of bunch halo by having the bunch tilted in the early part of the cycle.

\section{SECOND STAGE (7.5 MHZ RF)}

In stage 2 the PD is used to produce $\mu$ 's for a $\nu$ factory storage ring. The extraction energy is raised to 16 $\mathrm{GeV}$ and the rf system is replaced with an $h=18$ system to provide the desired bunch spacing. A factor four larger extracted longitudinal emittance is allowed for each of the 18 bunches, so the design brightness is raised by only $65 \%$. The larger inter-bunch gap permits chopping the linac beam, allowing synchronous injection. The linac beam spans $252^{\circ}$ of an approximately stationary bucket. There is an additional requirement for $<3 \mathrm{~ns}$ rms bunch length at extraction. It can be met by keeping the voltage at 1.4 MV as $\dot{B}$ drops toward the end of the acceleration cycle. The rms bunch length is $0.64 \mathrm{~ns}$ with a bunch rotation and $1.55 \mathrm{~ns}$ without. The final $95 \%$ emittances are $0.43 \mathrm{eVs}$ and $0.39 \mathrm{eVs}$ respectively. The rf parameters of PD stage 2 are collected in Table 1.

\subsection{Stage 2 RF Curves}

Because the beam is chopped and there is more adequate rf focusing in stage 2 , an inductive insert is not used. There are practically no losses, not only at injection, but throughout the acceleration cycle. The voltage and magnetic ramp curves are similar to those found for stage 1, but the buckets are less full and there is no need for fine tuning of the curves to control losses.

For the narrowest bunches a bunch rotation is intended. However, merely keeping the voltage at its maximum permissable value of 1.4 MV until the end of the cycle gives al-

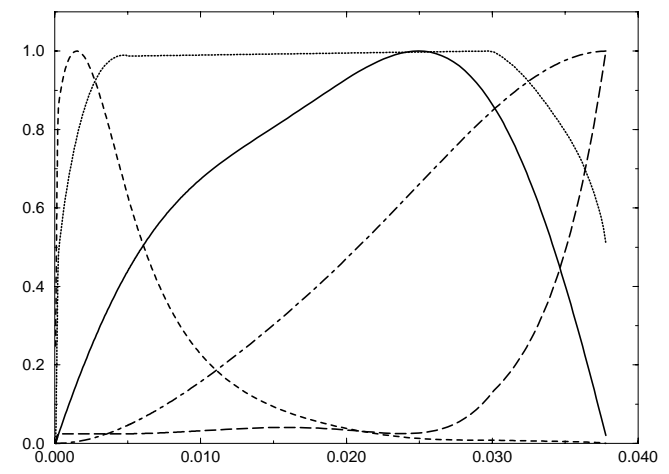

Figure 1: RF parameters durning the cycle of the Stage 1 proton driver scaled to the range 0 to $1: V_{\mathrm{rf}}$ (fine dots), $p$ (dash-dot), $\dot{p}$ (solid), $\nu_{\mathrm{s}}$ (short dash), and bucket area (long dash)

ready an rms bunch length of $1.55 \mathrm{~ns}$, somewhat better than had been anticipated in the initial design. For injection into the Main Injector the final voltage can be set at any convenient value between there and $100 \mathrm{kV}$ or so. Even narrower bunches can be obtained by a quarter period bunch rotation in a mis-matched bucket. The momentum spread becomes wide enough that the contribution of the second and perhaps the third order dependence of path length on momentum are important. These contributions are included in the macroparticle model. Considered but not included here is the effect of path length difference depending on betatron amplitude. Figure 1 shows the phase space distribution of a $0.39 \mathrm{eVs}$ bunch at extraction without rotation in a bucket produced by the maximum $1.4 \mathrm{MV}$ of rf. If a rotation is made, it starts at $37.6 \mathrm{~ms}$ when the sychronous phase is $\phi_{s}=70^{\circ}$ and $V_{\mathrm{rf}}$ is $145 \mathrm{kV}$. Fig. 3 shows the phase space distribution; it has an rms emittance of $0.43 \mathrm{eVs}$ and rms length of $0.64 \mathrm{~ns}$.

\section{REFERENCES}

[1] “The Proton Driver Design Study”, Fermilab TM-2136 (December 2000)

[2] M. Champion, T. Berenc, M. May, and J. Reid, "Design and Prototype Tests of a Large Aperture 37-53 MHz Ferrite Tuned Booster Cavity", this conf.

[3] J. Dey, I Kourbanis, Z. Qian and D. Wildman, "A Prototype 7.5 MHz Finemet Loaded RF Cavity and 200 MW Amplifier for the Fermilab Proton Driver", this conf.

[4] A. M. Sessler and V. G. Vaccaro, "Passive Compensation of Longitudinal Space Charge Effects in Circular Accelerators — The Helical Insert", CERN 68-1, ISR Div. (1968)

[5] K. Koba et al., "Longitudinal Impedance Tuner Using High Permiability Material”, KEK Preprint 99-96 (1999), also PAC99, New York

[6] M. A. Plum et al., "Experimental Study of Passive Compensation of Space Charge at the LANL Proton Storage Ring", Phys. Rev. ST Acc. and Beams, v. 2, 064201 (1999) 
Table 1: Proton Driver rf parameters

\begin{tabular}{|c|c|}
\hline \multicolumn{2}{|l|}{ General parameters } \\
\hline injection kinetic energy [MeV] & 400 \\
\hline beam intensity $[\mathrm{p} / \mathrm{cycle}]$ & $3 \cdot 10^{13}$ \\
\hline cycle repetition rate $[\mathrm{Hz}]$ & 15 \\
\hline circumference $/ 2 \pi[\mathrm{m}]$ & 113.21 \\
\hline energy spread at injection [MeV] & \pm 0.5 \\
\hline momentum compaction & $-1.306 \cdot 10^{-3}$ \\
\hline coefficient of $(\Delta p / p)^{2}$ in path & $8.252 \cdot 10^{-2}$ \\
\hline coefficient of $(\Delta p / p)^{3}$ in path & -0.4456 \\
\hline momentum acceptance [\%] & 2.5 \\
\hline vacuum chamber radius $[\mathrm{cm}]$ & 6.35 \\
\hline mean beam radius at injection $[\mathrm{cm}]$ & 4.44 \\
\hline \multicolumn{2}{|l|}{ Stage 1 parameters } \\
\hline extraction kinetic energy [GeV] & 12 \\
\hline maximum rf voltage [MV] & 1.2 \\
\hline accelerating voltage at $\dot{p}_{\max }[\mathrm{MV}]$ & 1.09 \\
\hline harmonic number & 126 \\
\hline number of populated buckets & 119 \\
\hline bunch intensity & $2.5 \cdot 10^{11}$ \\
\hline final rms emittance $[\mathrm{eVs}]$ & 0.1 \\
\hline \multicolumn{2}{|l|}{ Stage 2 parameters } \\
\hline extraction kinetic energy $[\mathrm{GeV}]$ & 16 \\
\hline maximum rf voltage [MV] & 1.4 \\
\hline accelerating voltage at $\dot{p}_{\max }[\mathrm{MV}]$ & 1.33 \\
\hline harmonic number & 18 \\
\hline bunch intensity & $1.7 \cdot 10^{12}$ \\
\hline final rms emittance $[\mathrm{eVs}]$ & 0.4 \\
\hline rms bunch length at extraction & $\leq 3$ \\
\hline
\end{tabular}

Table 2: Comparison of RMS emittance at extraction and fractional beam loss for optimum stage 1 parameters and cases differing each in a single property

\begin{tabular}{|l|cc|}
\hline Parameter set & emittance [eVs] & loss [\%] \\
\hline optimum set & 0.0197 & 0.03 \\
rf in two sets & 0.0181 & 0.07 \\
all rf clumped & 0.0154 & 0.21 \\
no inductive insert & 0.0247 & 3.19 \\
\hline
\end{tabular}

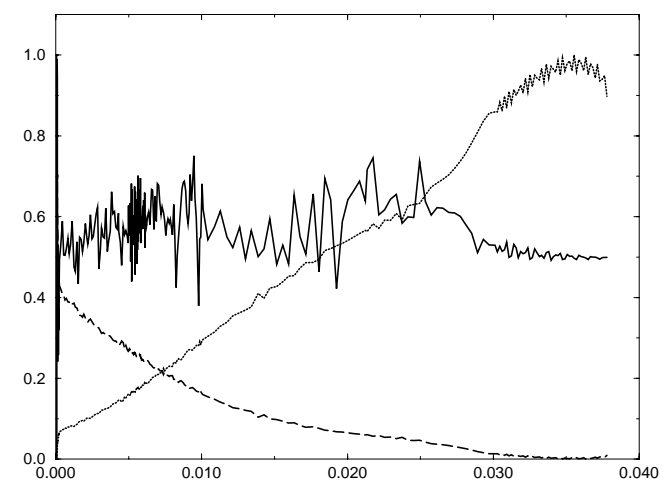

Figure 2: RMS normalized emittance (solid), rms bunch width (dashes), and rms bunch height (fine dots) for the stage one acceleration cycle, all scaled to plot in the range 0 to 1 .

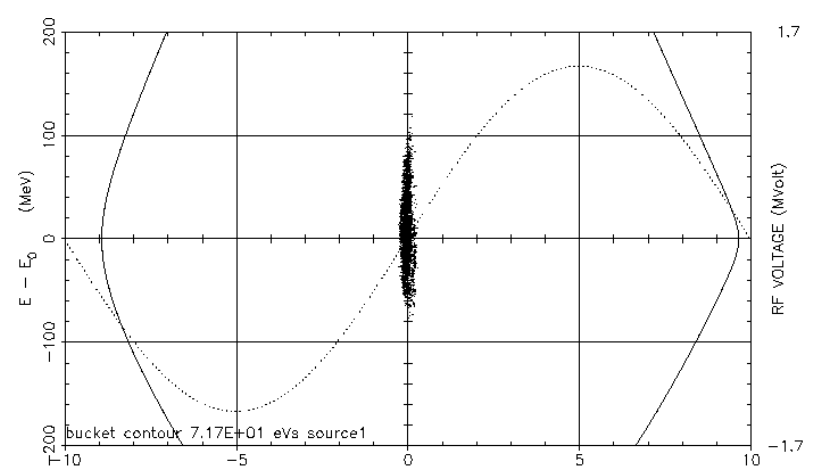

Figure 3: Energy $[\mathrm{MeV}] v s$. azimuth [deg] distribution of $.43 \mathrm{eVs}$ bunch of $1.7 \cdot 10^{12}$ protons at $16 \mathrm{GeV}$ after quarter period rotation at $1.4 \mathrm{MV}$, Stage 2 Proton Driver.

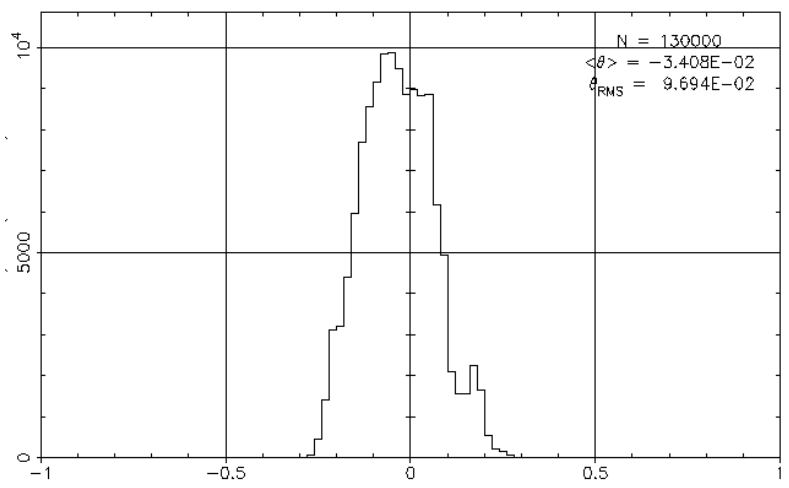

Figure 4: Azimuthal projection of roatated bunch shown in Fig. 3, abcissa in degrees. The rms bunch length in time is $0.64 \mathrm{~ns}$. 